\title{
Separation of small targets in multi- wavelength mixtures based on statistical independence
}

Rami Mowakeaa, Darren K. Emge

Rami Mowakeaa, Darren K. Emge, "Separation of small targets in multiwavelength mixtures based on statistical independence," Proc. SPIE 10646, Signal Processing, Sensor/Information Fusion, and Target Recognition XXVII, 106461H (8 June 2018); doi: 10.1117/12.2305061

SPIE. Event: SPIE Defense + Security, 2018, Orlando, Florida, United States 


\title{
Separation of Small Targets in Multi-Wavelength Mixtures Based on Statistical Independence
}

\author{
Rami Mowakeaa ${ }^{\mathrm{a}}$ and Darren K. Emge ${ }^{\mathrm{b}}$ \\ ${ }^{a}$ University of Maryland, Baltimore County, 1000 Hilltop Circle, Baltimore, USA \\ ${ }^{\mathrm{b}}$ US Army, Edgewood Chemical Biological Center, RDCB-DRC-P, APG, MD, USA
}

\begin{abstract}
Small target detection is a problem common to a diverse number of fields such as radar, remote sensing, and infrared imaging. In this paper, we consider the application of feature extraction for detection of small hazardous materials in multiwavelength imaging. Since various materials may exist in the area of study each with varying degrees of reflectivity and absortion at different wavelengths of light, flexible, data-driven methods are needed for feature extraction of relevant sources. We propose the use of independent component analysis (ICA), a widely-used blind source separation method based on the statistical independence of the underlying sources. We compare 3 different prominent flavors of ICA on simulated data in a variety of environments. Then, we apply ICA to 2 multi-wavelength imaging datasets with results that suggest that features extracted are useful.
\end{abstract}

Keywords: Small-target detection, ICA, BSS, multi-wavelength, machine learning

\section{INTRODUCTION}

Techniques for detection of malicious hazardous materials in otherwise benign environments have generated close attention lately with the growth of global security concerns ${ }^{1}$ as well as safety applications involving remote sensing, ${ }^{2}$ infrared (IR) imaging ${ }^{3}$ and others. In multi-wavelength imaging, a surface of interest is scanned by a tunable, variable-wavelength laser or a diffractive/dispersive instrument separating broadband illumination, producing complimentary images of the same surface at different wavelengths with the purpose of detecting malicious or hazardous materials. In many multi-wavelength imaging applications, the target material is not known in advance, limiting the performance of model-based approaches. Furthermore, the target may occupy an exceedingly small proportion of the area under investigation which can overwhelm methods that process full-resolution images. Therefore, there is a need for data-driven feature extraction to extract sensible signals of reduced dimensionality as a preprocessing step. ${ }^{4}$ In this paper, we propose the use of principle component analysis (PCA) to reduce the set of images followed by one of 3 popular independent component analysis (ICA) methods to separate a set of features from the residual mixtures. ICA has been widely used in fields such as medical imaging, ${ }^{5}$ communications, ${ }^{6}$ and acoustic data analyses. ${ }^{7}$ We apply this approach to a number of simulated examples and highlight the performance of each. Then, we show the result of applying this technique to 2 datasets acquired from a multi-wavelength, tunable laser applied to a test surface containing sample targets.

The remainder of this paper is organized as follows: Section 2 provides background for the proposed methods. In Sec. 3, we detail the experimental design and real-world dataset and discuss the results. Finally, we summarize our conlusions in Sec. 4.

\section{MATERIALS AND METHODS}

Blind source separation (BSS) methods attempt to solve the problem of identifying latent, or unobservable, sources given a set of observations where neither the sources, nor the generative model of the mixtures, is known. In particular, ICA, has been extensively used when the assumption of statistical independence of the latent sources is reasonable and little other prior information about the sources is known.

Further author information: (Send correspondence to D.K.E)

D.K.E.: E-mail: darren.k.emge.civ@mail.mil , Telephone: 14104366627

R.M.: E-mail: ramo1@umbc.edu, Telephone: 14104551000

Signal Processing, Sensor/Information Fusion, and Target Recognition XXVII, edited by Ivan Kadar,

Proc. of SPIE Vol. 10646, 106461H · C 2018 SPIE · CCC code: 0277-786X/18/\$18 · doi: 10.1117/12.2305061 


\subsection{Images as Sources}

Given an image $\tilde{\mathbf{X}} \in \mathbb{R}^{W \times L}$, we view each pixel as a realization of a random variable by serializing its entries in a vector, i.e., $\mathbf{X} \in \mathbb{R}^{V \times 1}$, where $V=W \times L$. In multi-wavelength imaging, we are given a collection of images $\left\{\mathbf{X}_{i}\right\}_{i=1}^{M}$ parameterized by $i$, where $i$ is the index of the wavelength at which the given image was collected. We collect these images in a single matrix $\mathbf{X}=\left[\mathbf{x}_{1}, \ldots, \mathbf{x}_{M}\right]^{\top}$, where $(\cdot)^{\top}$ denotes the transpose. Each of these images is then viewed as a mixture of $N \leq M$ statistically independent sources. Usually, we assume that there is an equal number of mixtures and sources. When $N<M$, a dimensionality reduction step can be performed on the mixtures using $\mathrm{PCA}^{8}$ with an order selected based on capturing most of the variance of mixtures.

\subsection{The ICA Model}

In this section, we provide a brief overview of the ICA problem and broad approaches used to solve it. We then summarize the differences between the algorithms used. For a detailed treatment of ICA, we refer the reader to Ref. 9 and references associated with each method.

In the noiseless ICA approach, a linear generative model is assumed as in:

$$
\mathbf{x}=\mathbf{A s},
$$

where $\mathbf{s} \in \mathbb{R}^{N}$ is the latent source random vector, $\mathbf{x}$ is a random vector containing the observations, and $\mathbf{A} \in$ $\mathbb{R}^{N \times N}$ is an invertible mixing matrix. Note that the entries $\left\{s_{i}\right\}_{i=1}^{N}$ are assumed to be statistically independent and both $\mathbf{s}$ and $\mathbf{A}$ are unknown. The goal of ICA is to estimate a demixing matrix $\mathbf{W}$ such that

$$
\mathbf{Y}=\mathbf{W X}
$$

and $\left\{\mathbf{y}_{i}\right\}_{i=1}^{N}$ are statistically independent, where $\mathbf{X}$ are the collection of images as in Sec. 2.1. Many approaches exist to solve for $\mathbf{Y}$ in Eq. (1). One approach involves maximizing the log-likelihood of the data given the observations as in: ${ }^{9}$

$$
\mathcal{L}(\mathbf{W} \mid \mathbf{X})=\sum_{v=1}^{V} \sum_{n=1}^{N} \log p_{i}\left(y_{i}(v)\right)+V \log |\operatorname{det}(\mathbf{W})|,
$$

where the importance of choosing appropriate source distributions $\left\{p_{n}(\cdot)\right\}_{n=1}^{N}$ is apparent. Another approach to solve Eq. 1 is to minimize the mutual information between $\mathbf{Y}$ and $\mathbf{X}$ which is defined by: ${ }^{9}$

$$
\mathcal{I}\left(y_{1}, \ldots, y_{N}\right)=\sum_{n=1}^{N} \mathcal{H}\left(y_{n}\right)-\mathcal{H}(\mathbf{x}),
$$

where $\mathcal{I}(\cdot)$ is the mutual information function and $\mathcal{H}(\cdot)$ is the differential entropy. It can be shown that both approaches are equivalent when the model distributions match the true latent distributions as $V \rightarrow \infty$.

Efficient FastICA (EFICA), models each underlying source distribution with a generalized Gaussian (GG) adaptively, through sequential estimation of the shape parameter for each source. ${ }^{10}$ EFICA begins by prewhitening the mixtures, then finds an orthogonal rotation matrix that produces independent sources estimates.

Infomax $^{11,12}$ maximizes the information transfer between the mixtures and estimated sources. It utilizes a nonlinear function that maps the real line to the range $[0,1]$ which is shown to capture higher-order moments of the estimated source signals without prior knowledge of the source distributions.

ICA-EBM uses 4 measuring functions to adapt to latent source distributions in a flexible, data-driven fashion. The authors show that these measuring functions can efficiently model source distributions that are sub-Gaussian, super-Gaussian, unimodal, bimodal, symmetric, and skewed PDFs. ${ }^{13}$ 


\section{EXPERIMENTAL RESULTS}

\subsection{Simulated Sources}

In this subsection, we apply the 3 ICA algorithms EFICA, Infomax, and ICA-EBM to a set of simulated mixtures to compare their separation power. In each simulation, we generate $N=3$ underlying source images. The first image, representing the background, consists of random noise generated from one of following 3 distributions: uniform, Laplacian, and Gaussian. In order to represent a number real-world scenarios, we consider a spatial distributions: constant, ramp, and step. In Fig. 1 we show each of the resulting 9 cases.

The next 2 underlying target sources are each modeled as spatially-circular with a randomly-positioned center of radius $r<l$, where $l=128$ is the length of each square image. Sample sources are displayed in Fig. 2

To generate the mixtures, we note that the $n^{\text {th }}$ column of the mixing matrix $A$ represents the reflected contribution of the $n^{\text {th }}$ source over different wavelengths. Inspired by data from real-world sources, we model each column by a mixture of $K$ Gaussian distributions, where $K \in\{3,4,5\}$. We show a number of sample mixing columns in Fig. 3, where the number of mixtures is $M=50$. In this section, the order of the underlying sources
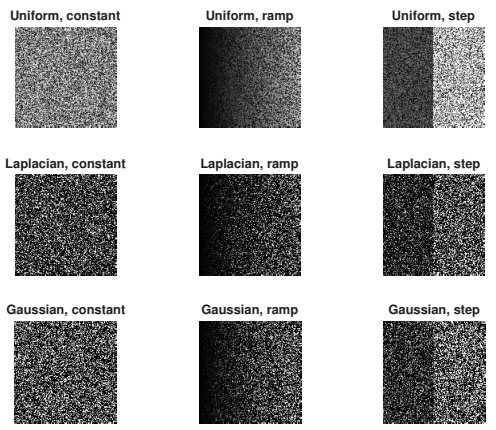

Figure 1. Background sources generated for each experiment. From left to right, the spatial distribution (constant, ramp, step). From top to bottom, the statistical distribution (uniform, Laplacian, Gaussian)

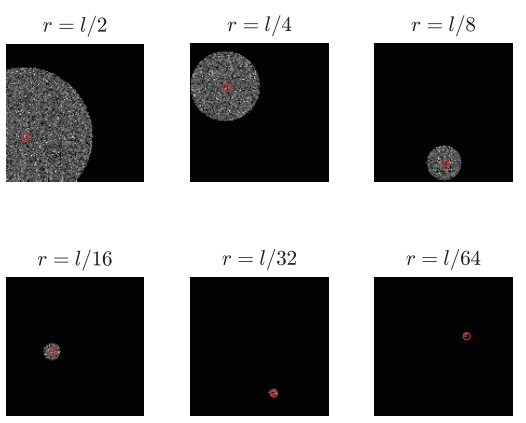

Figure 2. Examples of generated target sources with different radii as a proportion of the length of the source image. The randomly-placed center of each source is shown in red.

is known $N=3$, and PCA is used to reduce the number of mixtures from $M$ to $N$. Then, each set of mixtures was processed using each of the ICA methods EFICA, Infomax, and ICA-EBM. To quantify the average quality of the decompositions, we use inter-symbol interference (ISI) ${ }^{14}$ defined by:

$$
\operatorname{ISI}(\mathbf{G})=\frac{1}{2 N(N-1)}\left[\sum_{n=1}^{N}\left(\sum_{m=1}^{N} \frac{\left|g_{n, m}\right|}{\max _{p}\left|g_{n, p}\right|}-1\right)+\sum_{m=1}^{N}\left(\sum_{n=1}^{N} \frac{\left|g_{n, m}\right|}{\max _{p}\left|g_{p, m}\right|}-1\right)\right],
$$




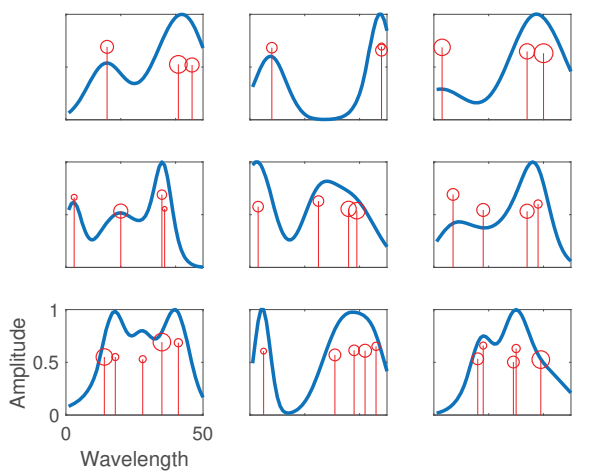

Figure 3. Sample mixing columns of $A$. For reference, the randomly selected mean, standard deviation, and amplitude, represented as the position, marker size, and amplitude of each constituent Gaussian is shown in red.

where $\mathbf{G}=\left[g_{n, m}\right]=$ WA. ISI measures the deviation of $\mathbf{G}$ from the identity matrix and is bound to the range $[0,1]$, with 0 implying perfect source estimation subject to the scaling and permutation ambiguity of ICA. The result of running each of these experiments are shown in Fig. 4 where each point is the result is the average of 100 independent runs and the standard deviation is shown as error bars. It is clear from Fig. 4, that the spatial

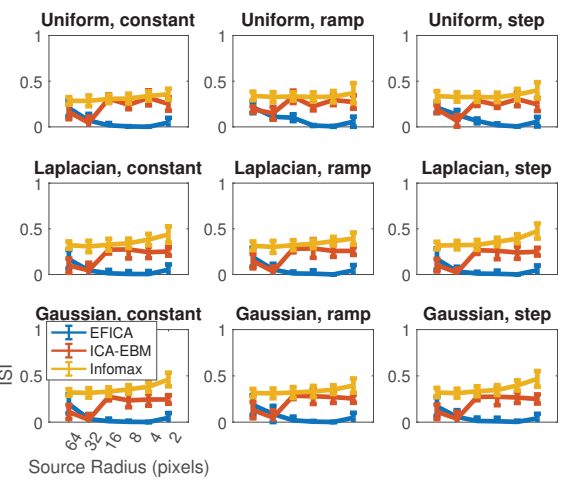

Figure 4. The average and standard deviation of the ISI resulting from running 100 independent runs over $r \in$ $\{2,4,8,16,32,64\}$ pixels in each of the 9 background environments considered.

distribution of the background plays no obvious role in the performance of each algorithm. This is precisely the motivation for using a BSS technique: by analyzing the mixtures in a statiscal manner, our approach is agnostic, and indifferent, to the spatial distribution of the sources within each image. Also, it is clear that Infomax does not model source distributions well for this application and its performance decreases with the decrease in source area. ICA-EBM, with its complex density estimation performs the best when sources are large, but performance suffers rapidly as the number of samples containing useful source information decreases. Finally, EFICA with its flexible, yet simple distribution model, continues to improve with decreasing source radius to a point after which its performance too begins to suffer. This initial behavior of EFICA is likely due to an increased chance of significant overlap between the target sources which can make separation more difficult.

\subsection{Real-World Dataset}

We consider a $75 \mathrm{~mm} \times 75 \mathrm{~mm}$ area of surface scanned by a flexible, compact, tunable laser illuminate IR imager. At each wavelength, the reflected energy is captured by an IR-sensitive camera. Each camera pixel then captures the scattered light from the combination of materials in the corresponding surface area. Two such datasets were collected where a material of interest was placed in a 9-dot square pattern in the center of the surface as shown in Fig. 5. In each of the 2 datasets, 22 mixtures were provided at wavelengths $(2700+k \times 50), k \in\{1, \ldots, 22\}$, 


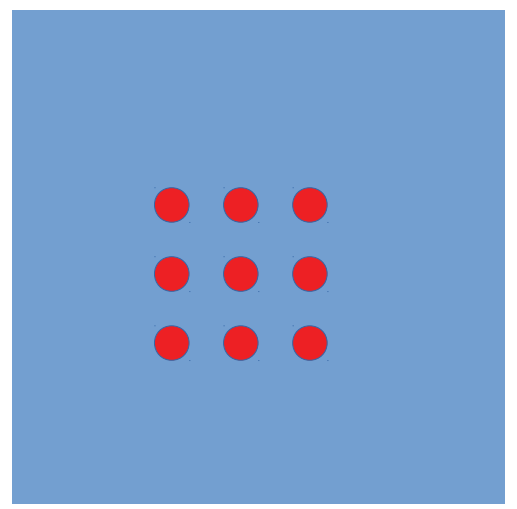

Figure 5. Experimental setup with 9 instances of a material of interest on the scan surface.
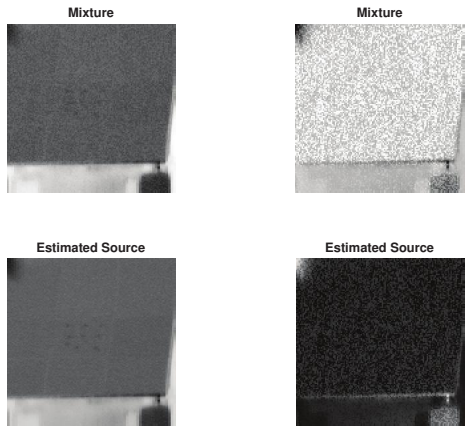

Figure 6. Dataset 1: The result of decomposing imaging mixtures using ICA. The top row shows the dimensionalityreduced mixtures. The bottom row shows the estimated underlying sources.

i.e., wavlengths $2700 \mathrm{~nm}$ through $3750 \mathrm{~nm}$ in steps of $50 \mathrm{~nm}$.

We determine the order for dimensionality reduction by traversing the ordered set of singular values of the mixutre matrix and stopping when the next singular value decreases by $80 \%$ from the current singular value. This consistently yielded an order of 2. After performing PCA on the mixture matrix with order 2, we apply EFICA, which performed best in our simulations for small targets, to produce 2 estimated sources. The results are shown in Figs. 6 and 7 where the top row shows the reduced input mixtures, i.e., the output of the PCA stage, and the bottom row shows the corresponding estimated sources. In both datasets PCA helps separate the background noise from the target image, but we see that the estimated sources represent further denoised features.

\section{CONCLUSION}

In this paper, we propose the use of ICA to extract latent features from multi-wavelength images blindly, based on the statistical independence of the sources. Our simulated experiments show that ICA is capable of extracting target sources with little regard to the spatial distribution of these sources. Our approach shows that EFICA, with a simple, yet flexible model is well-suited to small-target feature extraction in both simulated, as well 2 real-world multi-wavelength imaging datasets. 


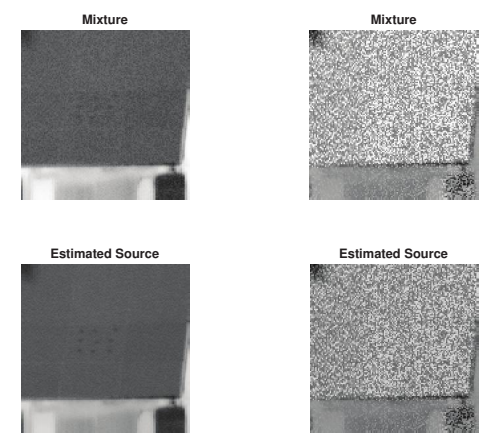

Figure 7. Dataset 2: The result of decomposing imaging mixtures using ICA. The top row shows the dimensionalityreduced mixtures. The bottom row shows the estimated underlying sources.

\section{REFERENCES}

[1] Tribe, W. R., Newnham, D. A., Taday, P. F., and Kemp, M. C., "Hidden object detection: security applications of terahertz technology," in [Terahertz and Gigahertz Electronics and Photonics III], 5354, 168-177, International Society for Optics and Photonics (2004).

[2] Gu, Y., Wang, C., Liu, B., and Zhang, Y., "A kernel-based nonparametric regression method for clutter removal in infrared small-target detection applications," IEEE Geoscience and Remote Sensing Letters 7(3), 469-473 (2010).

[3] Bai, X. and Zhou, F., "Analysis of new top-hat transformation and the application for infrared dim small target detection," Pattern Recognition 43(6), 2145-2156 (2010).

[4] Wang, Y., Lan, Q., and Zhang, T., "Target detection based on hierarchical saliency feature model," in [MIPPR 200\%: Automatic Target Recognition and Image Analysis; and Multispectral Image Acquisition], 6786, 67863H, International Society for Optics and Photonics (2007).

[5] Rodriguez, P. A., Correa, N. M., Eichele, T., Calhoun, V. D., and Adalı, T., "Quality map thresholding for de-noising of complex-valued fMRI data and its application to ICA of fMRI," Journal of Signal Processing Systems 65(3), 497-508 (2011).

[6] Lu, G., Xiao, M., Wei, P., and Li, J., "Single channel blind separation of oversampling communication signals based on ICA," in [Communication Problem-Solving (ICCP), 2014 IEEE International Conference on], 364-367, IEEE (2014).

[7] Katoozian, D. and Faradji, F., "Singer's voice elimination from stereophonic pop music using ica," in [Intelligent Systems and Signal Processing (ICSPIS), 2017 3rd Iranian Conference on], 174-177, IEEE (2017).

[8] Hyvärinen, L., "Principal component analysis," in [Mathematical Modeling for Industrial Processes], 82-104, Springer (1970).

[9] Hyvärinen, A., Karhunen, J., and Oja, E., [Independent component analysis], vol. 46, John Wiley \& Sons (2004).

[10] Koldovsky, Z., Tichavsky, P., and Oja, E., "Efficient variant of algorithm fastica for independent component analysis attaining the cramér-rao lower bound," IEEE Transactions on neural networks 17(5), 1265-1277 (2006).

[11] Bell, A. J. and Sejnowski, T. J., "An information-maximization approach to blind separation and blind deconvolution," Neural Computation 7(6), 1129-1159 (1995).

[12] Cardoso, J.-F., "Infomax and maximum likelihood for blind source separation," IEEE Signal processing letters 4(4), 112-114 (1997).

[13] Li, X.-L. and Adalı, T., "Independent component analysis by entropy bound minimization," IEEE Transactions on Signal Processing 58(10), 5151-5164 (2010).

[14] Moreau, E. and Macchi, O., "A one stage self-adaptive algorithm for source separation," in [Acoustics, Speech, and Signal Processing, 1994. ICASSP-94., 1994 IEEE International Conference on], 3, III-49, IEEE (1994). 